\title{
Attack organization
}

\section{During the onset of an attack, a subpopulation of these neurons showed their peak firing rates}

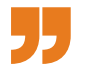

The ventrolateral part of the ventromedial hypothalamus ( $\mathrm{VMHvl}$ ) is a key region involved in generating aggression towards conspecifics, but how VMHvl neurons organize such hostile behaviour is not clear. Now, Falkner et al. describe a circuit in mice, including neurons in the VMHvl and the lateral periaqueductal grey (lPAG), that transforms social information into action-specific neural activity that is temporally aligned with biting in an attack.

VMHvl neurons can show activity when mice perform sensory investigations of conspecifics, prepare for aggressive action and conduct an attack, indicating that the VMHvl has a complicated role in conspecific aggression. To further understand its role, Falkner et al. first investigated potential functional connections between the VMHvl and the IPAG, as the IPAG receives projections from the VMHvl and the PAG has been implicated previously in aggression.

The authors used an optogenetic approach to stimulate excitatory VMHvl neurons while recording IPAG neurons in slices. Light stimulation led to 1 PAG neurons expressing a glutamatergic marker

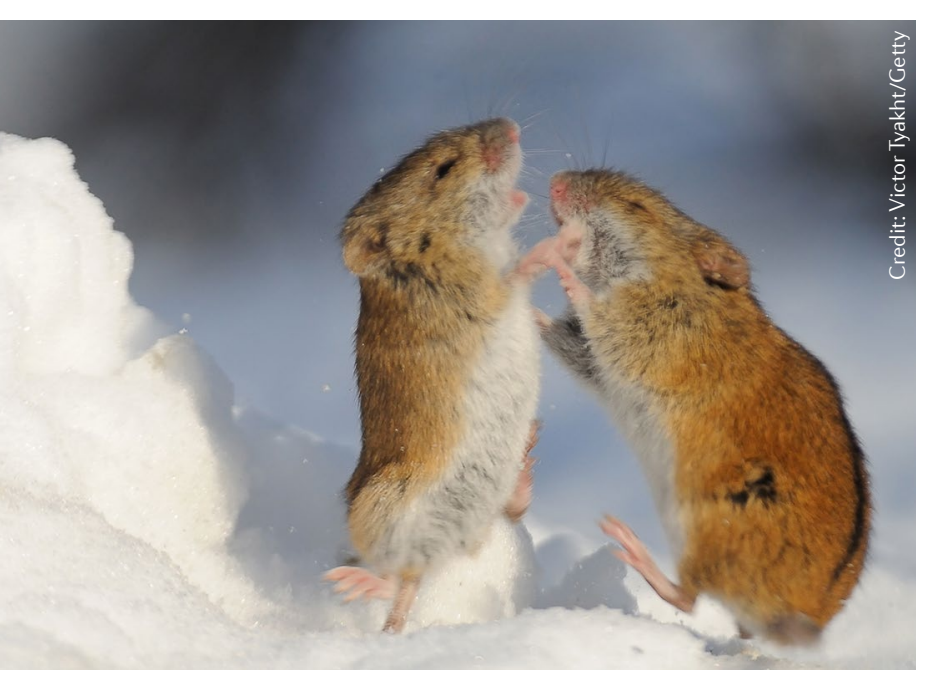

to exhibit short-latency excitatory postsynaptic currents (EPSCs), indicating a connection between the VMHvl and IPAG.

Biting is a key component of aggressive acts in mice, and the PAG sends projections to jaw muscles. The authors used pseudorabies virus-mediated retrograde tracing to examine whether IPAG neurons functionally connect to such muscles. Injection of the virus into the superficial masseter muscle, involved in jaw closure, led mostly to labelling of glutamatergic IPAG neurons. By combining the optogenetic and retrograde tracing approaches, the authors also showed that light stimulation of glutamatergic VMHvl neurons induced EPSCs in just over $50 \%$ of retrogradely labelled $\mathrm{IPAG}$ neurons. Together, these findings reveal a circuit by which the VMHvl is connected to the jaw muscles.

Stimulation of VMHvl cell bodies is sufficient to generate an attack response in a castrated male mouse. Here, optogenetic stimulation of the VMHvl-lPAG pathway increased the probability of such an attack in some animals, but it could not reliably generate such behaviour. Thus, the IPAG may have a role in organizing a specific motor component of an attack, in contrast to the broader role of the VMHvl in generating attack-related behaviours.

In line with this, male mice in which glutamatergic IPAG neurons were chemogenetically inactivated showed a decrease in the time they spent attacking during interactions with other male mice but no change in the time they spent investigating male or female animals or engaging in sexual behaviour.

The authors reasoned that this specific aggression-related deficit suggested that the IPAG neurons are involved in action selection.
To examine this, the authors recorded IPAG neurons while mice engaged in social interactions. During the onset of an attack, a subpopulation of these neurons showed their peak firing rates. By contrast, the investigation of male or female mice was associated with peak firing rates in few IPAG neurons. The VMHvl showed peaks in activities aligned to the onset of all three behaviours, indicating that $\mathrm{PAG}$ neural activity is more attack-selective than that in the VMHvl.

The authors examined whether some of the glutamatergic $1 \mathrm{PAG}$ neurons that receive VMHvl inputs coordinate muscle activity relevant to attacks. By simultaneously recording activity in the superficial masseter and IPAG neurons while mice engaged in attacks and other social interactions, they found that a subpopulation of these neurons showed altered activity during attack-linked movement of the jaw. Indeed, there was a strong temporal relationship between the activity of jaw muscles during biting and that of some lPAG neurons.

The authors next used fibre photometry to compare the activity of all glutamatergic VMHvl neurons with that of the glutamatergic VMHvl-IPAG neurons while mice engaged in social interactions. The activity in both populations was not markedly different during interactions - investigations and attacks - with males, but the activity in VMHvl-1PAG neurons was lower than that in the larger neuronal population during interactions with females. This supports the idea that the VMHvl conveys a male-biased signal to the IPAG to initiate action selection.

Together, these findings describe a circuit through which the VMHvl may organize an attack.

Darran Yates

ORIGINAL ARTICLE Falkner, A. L. et al. Hierarchical representations of aggression in a hypothalamicmidbrain circuit. Neuron https://doi.org/10.1016/ j.neuron.2020.02.014 (2020) 